\title{
ASOCIACIÓN ENTRE LA INCIDENCIA DE LEISHMANIOSIS CUTÁNEA Y EL ÍNDICE DE DESARROLLO HUMANO Y SUS COMPONENTES EN CUATRO ESTADOS ENDÉMICOS DE VENEZUELA*
}

\author{
Alfonso J. Rodríguez-Morales , $^{1,2,3,4, a}$, Yuliana Pascual-González ${ }^{1,3, b}$, Jesús A. Benítez ${ }^{5, a}$, María A. \\ López-Zambrano ${ }^{6, c}$, Rosane Harter-Griep ${ }^{7, c}$, Luz M. Vilca-Yengle ${ }^{8,9, a}$, Rocío Cárdenas ${ }^{2,10, d}$
}

\begin{abstract}
RESUMEN
Objetivos. Evaluar las posibles asociaciones entre el índice de desarrollo humano (IDH) y sus componentes, y la incidencia de Leishmaniosis cutánea (LC) en cuatro estados endémicos de Venezuela (Mérida, Trujillo, Lara y Sucre) en el período 1994 al 2003. Materiales y métodos. La data socioeconómica (clasificada de acuerdo al Banco Mundial) se obtuvo del Instituto Nacional de Estadística y la epidemiológica del Ministerio de Salud, ambos de Venezuela. Para este estudio ecológico se evaluó la variación anual de las variables y se realizó modelos de regresión. Resultados. El IDH varió en el período, de 0,6746 en 1994 a 0,8144 en 2003 ( $p=0,90$ ), asimismo, se observó un aumento de la incidencia acumulada de Leishmaniosis, en especial del año 1998 (7,3 casos/100 000 hab) a 1999 (11,3 casos/100 000 hab). Al analizar con los modelos de regresión lineal, se observó que la relación entre las variables epidemiológicas y sociales era diferente a nivel de los Estados evaluados. Para Mérida y Trujillo se observó un descenso significativo de la incidencia de LC con relación al aumento del porcentaje de alfabetización $(p<0,05)$, de estudiantes matriculados $(p<0,05)$, la esperanza de vida $(p<0,05)$, su ingreso en USD per cápita por año $(p<0,05)$ e IDH $(p<0,05)$. Conclusión. Esta información refleja la influencia significativa de los indicadores socioeconómicos sobre la incidencia de la LC en los Estados Trujillo y Mérida, siendo inversa entre ambos tipos de variables; con el incremento o mejoría de los indicadores socioeconómicos, la incidencia acumulada de la enfermedad disminuyó.
\end{abstract}

Palabras clave: Leishmaniosis cutánea; Desarrollo social; Factores sociales; Economía; Educación (fuente: DeCS BIREME).

\section{ASSOCIATION BETWEEN CUTANEOUS LEISHMANIASIS INCIDENCE AND THE HUMAN DEVELOPMENT INDEX AND ITS COMPONENTS IN FOUR ENDEMIC STATES OF VENEZUELA}

\begin{abstract}
Objectives. Assess potential relationships between the Human Development Index (HDI) and its components and the incidence of cutaneous leishmaniasis (CL) in four endemic States of Venezuela (Mérida, Trujillo, Lara and Sucre) in the period 1994-2003. Material and methods. Socioeconomical data (classified according the World Bank) was obtained from the National Institute of Statistics, and the epidemiological data from the Ministry of Health, both from Venezuela. For this ecological study the annual variation of the variables was assessed and also regression models were done. Results. The HDI varied in the period from 0.6746 in 1994 to 0.8144 in 2003 ( $p=0.90$ ). During this time an increase in the cumulative incidence of Leishmaniasis was observed, particularly from 1998 (7.3 cases/100,000 pop) to 1999 (11.3 cases/100,000 pop). Analyzing the linear regression models, it was observed that the relationship between epidemiological and social variables was different at States levels. For Mérida and Trujillo it was observed a significant decrease in the CL regard to the increase of literacy $(p<0.05)$, of the gross combined enrollment $(p<0.05)$, life expectancy $(p<0.05)$, money income $(p<0.05)$ and the HDI $(p<0.05)$. Conclusion. This information reflects the significant influence of socioeconomical indicators on the CL incidence at Trujillo and Merida, being an inverse relationship between both types of variables; with an increase or improvement in the socioeconomical indicators, the disease incidence rate decreased.
\end{abstract}

Key words: Cutaneous leishmaniasis; Social development; Social factors; Economy; Education (source: MeSH NLM).

\footnotetext{
Sección de Inmunoparasitología, Instituto de Medicina Tropical Felix Pifano, Universidad Central de Venezuela. Caracas, Venezuela.

Instituto Experimental José Witremundo Torrealba, Núcleo Universitario Rafael Rangel, Universidad de Los Andes. Trujillo, Venezuela.

3 Cátedra de Salud Pública, Departamento de Medicina Preventiva y Social, Escuela de Medicina Luis Razetti, Universidad Central de Venezuela. Caracas, Venezuela.

4 Dirección de Estudios Poblacionales, Fundación Centro de Estudios sobre Crecimiento y Desarrollo de la Población Venezolana (FUNDACREDESA), Ministerio del Poder Popular para las Comunas. Caracas, Venezuela.

5 Dirección General de Salud Ambiental y Contraloría Sanitaria, Ministerio del Poder Popular para la Salud. Maracay, Venezuela.

6 Unidad de Políticas Públicas, Universidad Simón Bolívar. Caracas, Venezuela.

Instituto Oswaldo Cruz (FIOCRUZ). Río de Janeiro, Brasil.

8 Sociedad Científica de San Fernando, Facultad de Medicina, Universidad Nacional Mayor de San Marcos. Lima, Perú

9 Servicio de Medicina Preventiva y Salud Pública, Hospital Universitario Vall d’Hebron. Barcelona, España.

10 Instituto Departamental de Salud de Norte de Santander. Cúcuta, Colombia.

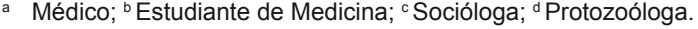

* Trabajo presentado en el 3rd Social Epidemiology Course, European Educational Programme of Epidemiology, Florencia, Italia, junio 2007.
}

Recibido: 27-04-09 Aprobado: 03-03-10 


\section{INTRODUCCIÓN}

Las poblaciones bajo el efecto de enfermedades endémicas enfrentan grandes obstáculos para lograr mejorar su calidad de vida. La Leishmaniosis cutánea (LC) constituye un ejemplo de una zoonosis que ha sido infravalorada en cuanto al efecto que produce sobre las comunidades en morbilidad, discapacidad y mortalidad. Simultáneamente, su situación epidemiológica - por los cambios climáticos, demográficos y socioeconómicos en las zonas tropicales del planeta - se ha hecho más compleja ${ }^{(1-3)}$.

La LC es una enfermedad tropical parasitaria metaxénica causada por especies del género Leishmania, siendo vectorizada en el continente americano por flebótomos del género Lutzomyia (1). A nivel epidemiológico, es importante destacar que la Leishmaniosis es una de las enfermedades con mayor carga en términos de discapacidad, estimada para el año 2003 a nivel mundial en 2,4 millones de años de vida ajustados por discapacidad (AVAD) ${ }^{(2)}$.

Al igual que muchas otras enfermedades infecciosas, tropicales, parasitarias y metaxénicas ${ }^{(2,3)}$, la LC puede verse influenciada por una serie de factores socioeconómicos capaces de incrementar o disminuir el número de casos propios o colindantes a una región, incluso su migración entre regiones endémicas (4) o a países y regiones no endémicas ${ }^{(5)}$. Sin embargo, los estudios que evalúen el impacto de las variables macroeconómicas y macrosociales sobre la incidencia de las enfermedades transmisibles han sido escasamente publicados ${ }^{(6)}$, más aun para el caso de la Leishmaniosis ${ }^{(7)}$.

Una forma de evaluar dichos impactos es analizando la influencia del Índice de Desarrollo Humano (IDH) sobre la epidemiología y carga de dichas enfermedades ${ }^{(6,7)}$. El IDH (del Programa de las Naciones Unidas para el Desarrollo) es usado para determinar e indicar si un país es desarrollado, subdesarrollado o se encuentra en vías de desarrollo, y también para medir el impacto de políticas económicas, sociales y sobre la calidad de vida ${ }^{(8)}$. El IDH es calculado como el promedio entre el índice de la esperanza de vida (LEI, life expectancy index), el índice de educación (El, education index) y el ingreso o índice del producto interno bruto $(\mathrm{PIB})^{(5,6)}$. El analfabetismo, el ingreso per cápita por año, la esperanza de vida en años y el acceso a educación constituyen las principales variables socioeconómicas directas que influyen en el IDH, el cual permite tener una visión instantánea del desempeño promedio nacional en materia de desarrollo humano ${ }^{(8)}$.

Actualmente existe una gran variación entre los países en relación con el grado desarrollo observado cuando se aplica el IDH, tal como se puede apreciar en la Figura 1, donde claramente se observa que aquellos con mayor desarrollo (alto, IDH $\geq 0,800$ ) se encuentran en Norteamérica y en Europa, en tanto que los de desarrollo medio (IDH 0,500-0,799) y bajo $(<0,500)$ están localizados en África, Asia y América Latina, particularmente en África subsahariana donde existe una gran cantidad de países con IDH $<0,500$ (Figura 1).

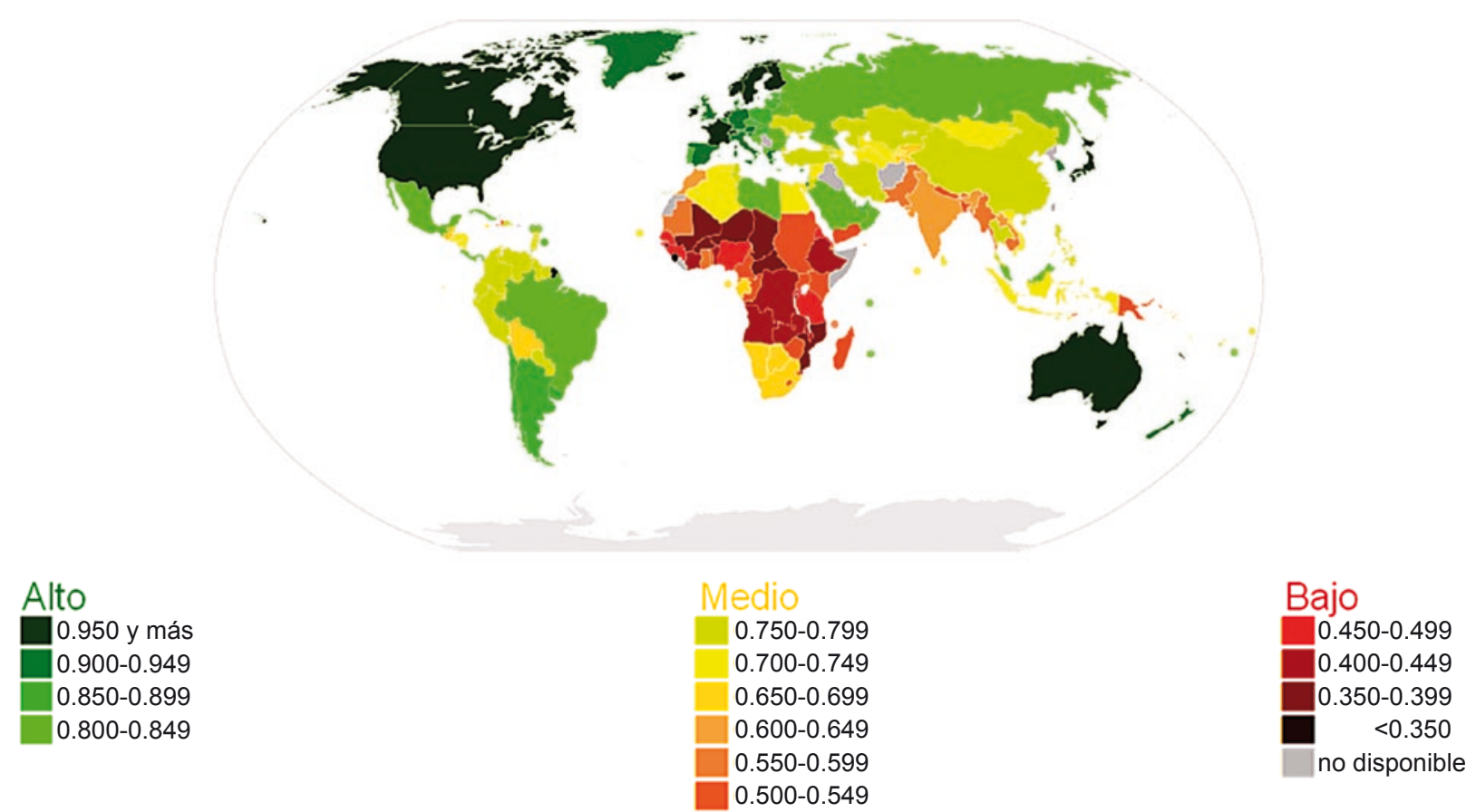

Figura 1. Mapa mundial indicando el IDH (2007) ${ }^{(8)}$.

Los países se clasifican en tres categorías en base a su IDH: alto, medio y bajo desarrollo humano. 
Son pocos los estudios que se han realizado sobre el IDH y su asociación con la incidencia de ciertas enfermedades, y ninguno en especial para Leishmaniosis cutánea $^{(6,7)}$.

En los Estados Mérida, Lara, Trujillo y Sucre tenemos que, en promedio, aproximadamente un niño de cada cinco abandona la escuela antes de completar el nivel de educación primaria (básica). No obstante, en algunos casos, las elevadas tasas de matrícula de niños que luego abandonan las clases ocultan el limitado progreso hacia la adquisición de las habilidades básicas de alfabetización; por lo cual es importante, además del estudio del índice de educación, el análisis de este indicador individualmente ${ }^{(4)}$. Por su parte, los ingresos y la esperanza de vida son indicadores fundamentales para el estudio de la situación socioeconómica, ergo, estos elementos deben evaluarse por separado y en el propio IDH integrado.

Este estudio se realizó con el objetivo de evaluar las posibles asociaciones entre el IDH y sus componentes, y la incidencia de Leishmaniosis cutánea (LC) en cuatro estados endémicos de Venezuela en el período 19942003.

\section{MATERIALES Y MÉTODOS}

\section{TIPO DE ESTUDIO}

Se llevó a cabo un estudio ecológico, valorando la asociación entre la incidencia de la LC y el IDH en cuatro estados de Venezuela.

\section{ÁREA GEOGRÁFICA DEL ESTUDIO}

Las áreas seleccionadas para el presente estudio fueron el Estado Mérida, el cual está ubicado al oeste del país; el Estado Trujillo, en el oeste del país; el Estado Lara, localizado en la zona centro-occidental del país; y por último el Estado Sucre, Venezuela ubicado al noreste del país (Figura 2).

Dichas regiones administrativas presentan gran variedad de ecosistemas, aunque corresponden desde un punto de vista general, para Mérida y Trujillo -ambos son estados andinos- a un ambiente de montaña con bosques de galerías de acuerdo con la clasificación de zonas de vidas de Holdridge; en el caso de Lara, que si bien tiene límite con Trujillo, se caracteriza por zonas áridas; y para Sucre, un ambiente de selva tropical lluviosa, con condiciones de costa con una gran cantidad de ríos pequeños atravesando los valles de poca pendiente y mal drenaje ${ }^{(3)}$.

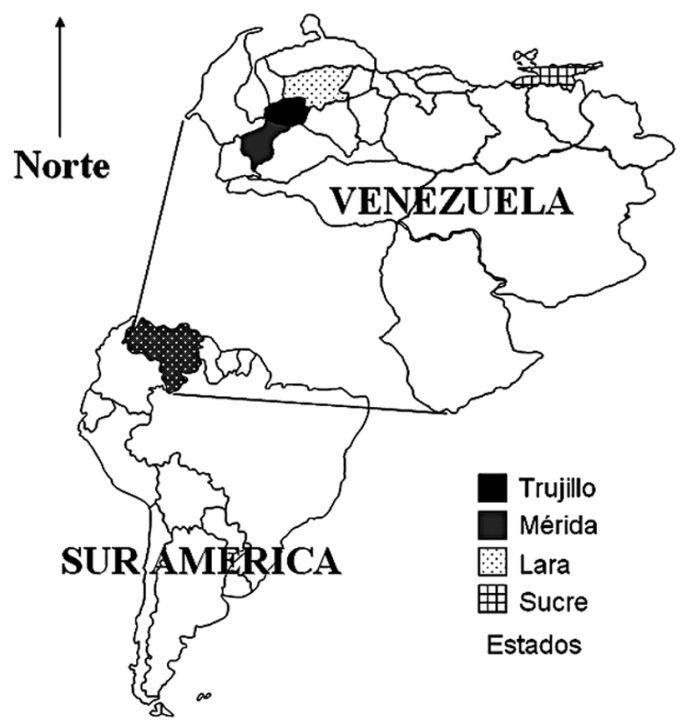

Figura 2. Mapa de Venezuela (destacando los estados evaluados).

\section{DEFINICIONES}

Los datos epidemiológicos para este estudio fueron obtenidos de los registros del Ministerio de Salud de Venezuela, con lo que fue realizado el análisis de morbilidad por Leishmaniosis cutánea en Venezuela durante el período de estudio. En este estudio se definió como caso de Leishmaniosis cutánea a todo aquel que fuese diagnosticado clínicamente, así como con al menos uno de los estudios paraclínicos realizados para confirmar el diagnóstico con resultado positivo (biopsia, cultivo, intradermorreacción, serología, reacción en cadena de polimerasa), los cuales son registrados inicialmente por la Unidad de Dermatología Sanitaria y obtenidos de la Dirección General de Salud Ambiental y Contraloría Sanitaria del Ministerio de Salud de Venezuela. La morbilidad registrada se dispuso en número de casos y en incidencia acumulada anual (casos/100 000 hab).

Por su parte, los datos socioeconómicos se obtuvieron del Instituto Nacional de Estadística, correspondiente al Ministerio de Planificación y Desarrollo de Venezuela, estos fueron: analfabetismo, ingreso, esperanza de vida, educación e IDH.

El IDH es calculado como el promedio entre el índice de la esperanza de vida (LEI), el índice de educación (EI) y el ingreso o índice del PIB (producto interno bruto) ${ }^{(5,6)}$. En general, para transformar una variable, llamada $x$, en un índice de unidad libre entre 0 y 1 (en el cual diferentes índices podrán ser sumados juntos), se utiliza la siguiente fórmula:

$$
x-\text { index }=(x-\min (x)) /(\operatorname{máx}(x)-\operatorname{mín}(x)) \text {; }
$$


Tabla 1. Valores del IDH y sus componentes medios, y de la incidencia acumulada medial anual de Leishmaniosis en algunos estados venezolanos endémicos, y a nivel nacional, 1994-2003.

\begin{tabular}{|c|c|c|c|c|c|c|}
\hline Estado & Alfabetismo & Ingreso & $\begin{array}{l}\text { Expectativa } \\
\text { de vida }\end{array}$ & Educación & IDH & $\begin{array}{l}\text { Incidencia acumulada } \\
\text { de Leishmaniosis* }\end{array}$ \\
\hline Trujillo** & 0,0221 & 0,0179 & 0,0027 & 0,0605 & 0,0189 & 46,83 \\
\hline Mérida** & 0,0063 & 0,0195 & 0,0271 & 0,0271 & 0,0226 & 33,26 \\
\hline Lara** $^{* *}$ & 0,2063 & 0,1087 & 0,0160 & 0,3022 & 0,2170 & 26,91 \\
\hline Sucre ${ }^{* *}$ & 0,1403 & 0,1461 & 0,5782 & 0,0950 & 0,0639 & 26,11 \\
\hline Yaracuy & 0,2669 & 0,0508 & 0,0999 & 0,2450 & 0,0502 & 20,88 \\
\hline Táchira & 0,2979 & 0,4772 & 0,8268 & 0,1789 & 0,3328 & 20,02 \\
\hline Miranda & 0,4907 & 0,3136 & 0,2238 & 0,6428 & 0,3085 & 11,85 \\
\hline Venezuela & 0,9274 & 0,9020 & 0,9752 & 0,7623 & 0,9020 & 10,28 \\
\hline
\end{tabular}

* media anual por 100 mil habitantes.

** Estados elegidos para el estudio.

Donde mín $(x)$ y máx $(x)$ son el mínimo y máximo valor que la variable $x$ puede tener, respectivamente. Entonces, el promedio es obtenido calculando el LEI, El y el índice del PIB para cada año.

La esperanza de vida $(\mathrm{LE})=(\mathrm{LE}-$ mínLE $) /($ máxLE mínLE ); el El = ( 2 / 3 ) x ALI + ( 1/ 3 ) x GEl; el índice de alfabetización de adultos $(\mathrm{ALI}$, adult literacy index $)=(A L R$ - 0 ) / ( $100-0$ ), donde ALR es la tasa de alfabetización de adultos ( $\%$ de $\geq 15$ años); la tasa neta de matriculación $(\mathrm{GEI}$, gross enrolment index $)=($ CGER -0$) /(100-$ 0 ), donde el CGER es el índice de matriculación bruta combinada en educación primaria, secundaria y terciaria (combined gross enrolment rate), y el índice PIB = [ $\log (\mathrm{PIBpc})$ - log (mínPIBpc) ] / [ log (máxPIBpc) - log (mínPIBpc) ], donde el PIBpc es el PIB per cápita en Paridad de Poder Adquisitivo (PPA) (PPP, purchasing power parity) en USD (dólar estadounidense).

Entonces, los valores mín(x) y máx(x) son obtenidos del UNDP (Programa de las Naciones Unidas para el Desarrollo) para cada año ${ }^{(7)}$. Por ejemplo, para el año 2003 en Venezuela, el "máximo" y "mínimo" de esperanza de vida fue de 85 y 25 años, respectivamente; mientras que el "máximo" y "mínimo" del PIB fue de 40000 y 100 USD (5). Para el índice de educación, el "máximo" y "mínimo" valor de la tasa de alfabetización y el CGER es de $100 \%$ y $0 \%$.

Finalmente, la fórmula para el IDH es: IDH = ( LEI + $\mathrm{EI}+$ PIBindex ) / 3. El valor del IDH indica que, si es $<0,5$ corresponde a un desarrollo humano bajo, entre 0,5 y 0,799 a un desarrollo humano medio $y \geq 0,8$ a un desarrollo humano alto.

\section{ANÁLISIS ESTADÍSTICO}

Se realizó primariamente una comparación cualitativa en las variaciones observadas en el analfabetismo (\%), ingreso (USD per cápita por año), esperanza de vida (años), educación e índice de desarrollo humano (IDH), con la incidencia de la Leishmaniosis cutánea (casos/100 000 hab) en los Estados Trujillo, Mérida, Lara y Sucre, durante el período seleccionado, y posteriormente correlaciones de Pearson entre dichas variables. Se realizaron regresiones lineales simples entre las variables epidemiológicas y socioeconómicas. Los análisis estadísticos se llevaron a cabo con los paquetes estadísticos SPSS v.10.0® y GraphPad Prism v.4® con un nivel de confianza de 95\% ( $p$ significativa $<0,05$ ).

\section{RESULTADOS}

Para el período de estudio, la incidencia acumulada de Leishmaniosis cutánea en Venezuela fue de 102,8 casos/100.000 hab, sin variaciones significativas de 1994 (12,1 casos/100.000 hab) a 2003 (11,5 casos/100.000 hab) $\left(r^{2}=0,45 ; p>0,05\right)$, pero con gran variación entre los diferentes estados del país (Tabla 1 ).

La incidencia acumulada media anual para el período estudiado fue de 10,28 casos/100.000 hab en Venezuela $\pm 1,568$ casos/100 000 hab $x$ año, ocupando Trujillo el primer lugar con una tasa de 46,83 casos/100 000 hab; seguido por Mérida con 33,26 casos/100 000 hab, Lara con 26,91 casos/100 000 hab y Sucre con 26,11 casos/100 000 hab (Figura 3).

Para este período, fue observado un incremento de la educación en Venezuela de 1994 (58,8\%) al 2003 $(75,0 \%) \quad\left(r^{2}=0,01, p=0,76\right) \quad$ (Figura $\left.3 A\right)$; también se observó para la esperanza de vida de 72 años en 1994 a 73,18 años en $2003\left(r^{2}=0,0001, p=0,97\right)$ (Figura 3B), el ingreso de 2100 USD per cápita en 1994 a 3924 USD per cápita en $2003\left(r^{2}=0,002, p=0,90\right)$ (Figura 3C) y la alfabetización de $88 \%$ en 1994 a $95 \%$ en 2003 $\left(r^{2}=0,001, p=0,93\right)$ (Figura 3D). El índice de desarrollo 

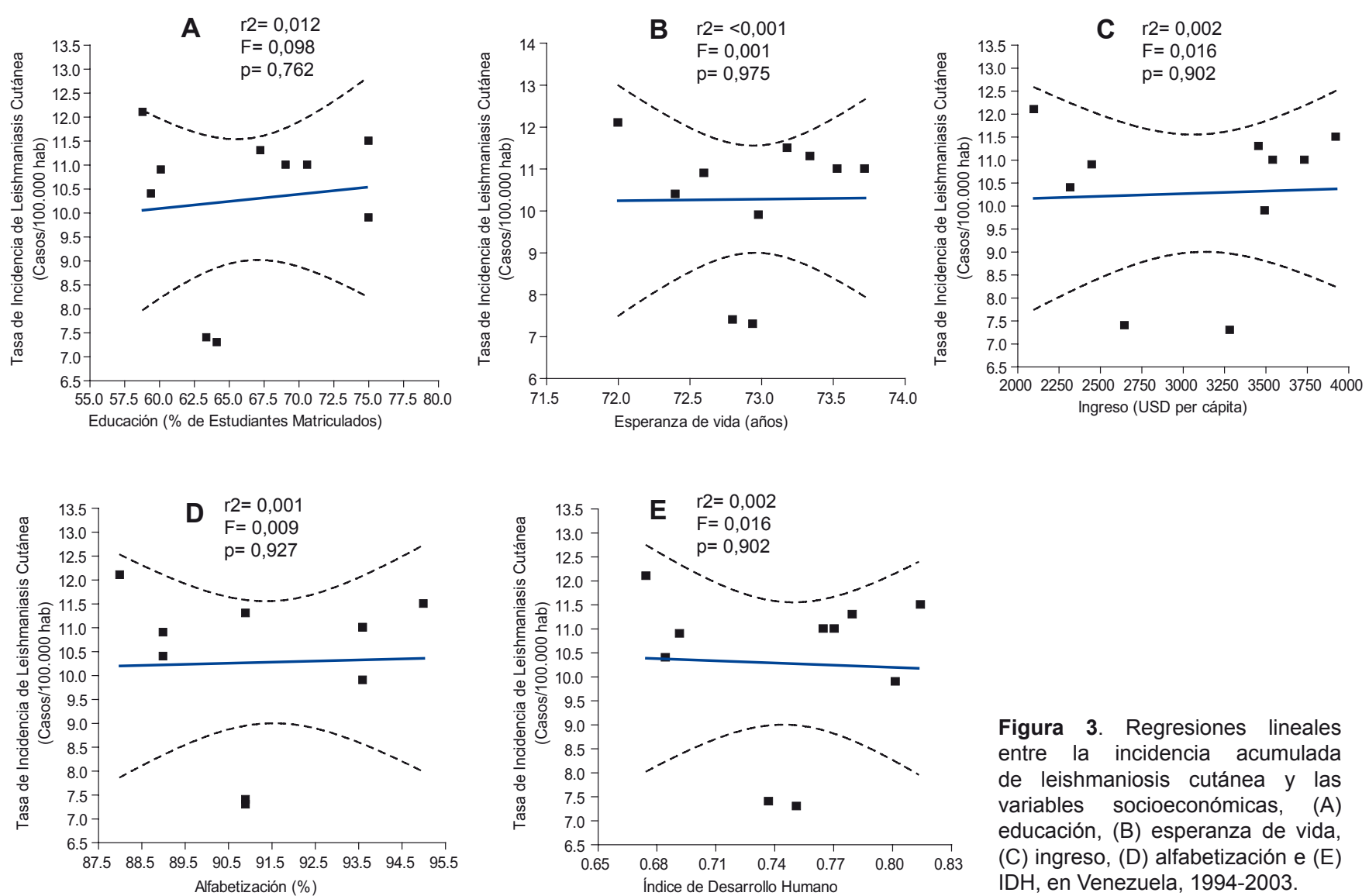

Figura 3. Regresiones lineales entre la incidencia acumulada de leishmaniosis cutánea y las variables socioeconómicas, (A) educación, (B) esperanza de vida, (C) ingreso, (D) alfabetización e (E) IDH, en Venezuela, 1994-2003.

humano (IDH) varió en el período, de 0,6746 en 1994 a 0,8144 en $2003\left(r^{2}=0,002, p=0,90\right)$ (Figura 3E). A pesar de los cambios ocurridos durante el período, al comparar simultáneamente los indicadores socioeconómicos y la tasa de incidencia de Leishmaniosis cutánea, pareciera no existir una influencia aparente en la relación a nivel nacional. A pesar de la mejora de las condiciones socioeconómicas del país se ha observado un aumento de la tasa de incidencia de Leishmaniosis, en especial del año 1998 (7,3 casos/100 000 hab) a 1999 (11,3 casos/100 000 hab).

Al analizar con los modelos de regresión lineal, se observó que la relación entre las variables epidemiológicas y sociales era diferente a nivel de los estados evaluados. Entre los años 1994-2003, Trujillo presentó un descenso significativo de la tasa de incidencia de Leishmaniosis cutánea con relación al aumento del porcentaje de alfabetización $\left(r^{2}=0,50, p=0,02\right)$, de estudiantes matriculados $\left(r^{2}=0,37, p=0,06\right)$, la esperanza de vida $\left(r^{2}=0,69, p=0,003\right)$, su ingreso en USD per cápita por año $\left(r^{2}=0,52, p=0,018\right)$ e índice de desarrollo humano $\left(r^{2}=0,52, p=0,019\right)$ (Figura 4).

Las regresiones lineales entre la morbilidad por Leishmaniosis cutánea y los indicadores socioeconómicos en el Estado Mérida (1994-2003), mostraron asociación significativa con la alfabetización $\left(r^{2}=0,63, p=0,006\right)$, educación $\left(r^{2}=0,48, p=0,025\right)$, esperanza de vida $\left(r^{2}=0,48, p=0,027\right)$, ingreso $\left(r^{2}=0,51\right.$, $p=0,02)$ e índice de desarrollo humano $\left(r^{2}=0,50\right.$, $p=0,023)$. Al igual que ocurre en Trujillo, en Mérida la relación es inversa entre ambas variables; cuando los indicadores socioeconómicos incrementan o mejoran, la tasa de incidencia de la enfermedad disminuye (Figura 5).

Para los Estados Lara y Sucre, ubicados respectivamente en el tercer y cuarto lugar, se observa que la relación entre las variables, morbilidad por Leishmaniosis cutánea y los indicadores socioeconómicos, no es significativa $(p>0,05)$, lo cual también es mostrado durante el mismo 

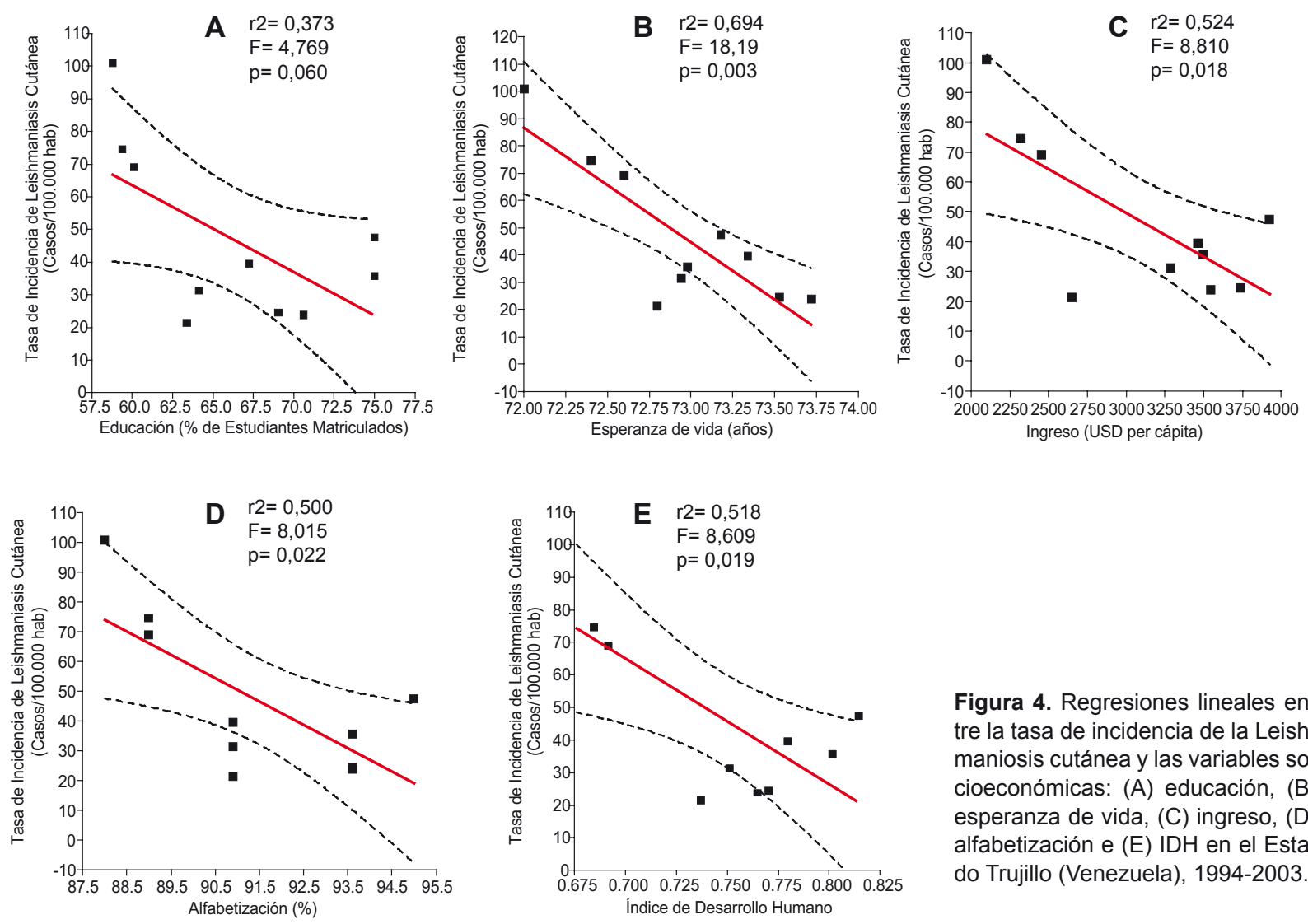

Figura 4. Regresiones lineales entre la tasa de incidencia de la Leishmaniosis cutánea y las variables socioeconómicas: $(A)$ educación, $(B)$ esperanza de vida, (C) ingreso, (D) alfabetización e (E) IDH en el Estado Trujillo (Venezuela), 1994-2003.
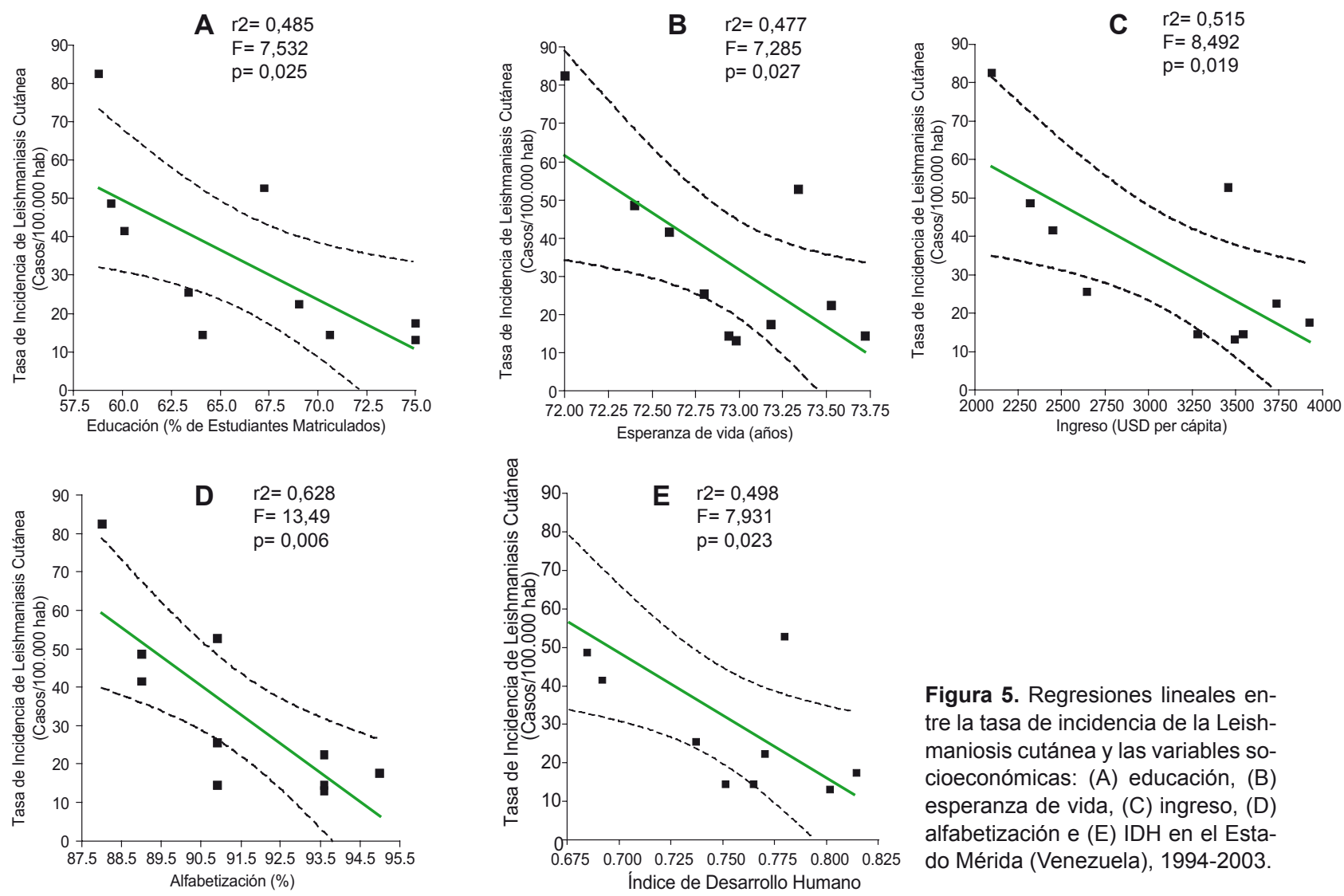

Figura 5. Regresiones lineales entre la tasa de incidencia de la Leishmaniosis cutánea y las variables socioeconómicas: $(A)$ educación, $(B)$ esperanza de vida, (C) ingreso, (D) alfabetización e (E) IDH en el Estado Mérida (Venezuela), 1994-2003. 
período en otros Estados adicionados posteriormente, pero que tienen una menor tasa de incidencia de la enfermedad (Yaracuy, Táchira, Miranda) (Tabla 1).

\section{DISCUSIÓN}

La asociación entre la morbilidad por Leishmaniosis cutánea y los indicadores socioeconómicos (educación, esperanza de vida, ingreso, alfabetización e IDH) entre los años 1994-2003, fue estadísticamente significativa para Trujillo y Mérida $(p<0,05)$, en comparación a las regresiones lineales obtenidas para los demás estados y a nivel nacional, donde ésta no fue significativa $(p>0,05)$.

Debemos mencionar que este estudio tiene limitaciones dadas por la naturaleza retrospectiva de los datos, el tipo de estudio (ecológico), así como, lo relacionado a incrementar la resolución temporal y espacial de los análisis, sumándose a ello la no inclusión de otras variables de interés en el análisis; esperándose mejorar en futuras investigaciones.

La relación inversa, significativa, encontrada entre ambos tipos de variables en los Estados Trujillo y Mérida se debe, entre otros factores, a la mayor incidencia acumulada de Leishmaniosis cutánea en la zona, por lo cual el número de casos fue representativo para su correlación. Sin embargo, en ambos estados se observa que la mejora en las condiciones socioeconómicas reflejadas en el IDH y sus componentes se asocia con una significativa reducción de la incidencia de la enfermedad. Esto pueden ser explicados por el mayor índice de migraciones con intención definitiva que presentan ambos Estados, debido a su cercanía con el noreste de Colombia, cuyos habitantes son forzados por situaciones de inestabilidad social - guerras, conflictos, persecución, entre otros- y económicas, a desplazarse fuera del país (12). A su vez, estos desplazamientos pueden conllevar a una gran limitación de las fuentes de ingreso de las familias afectadas, empeorando las condiciones de alimentación y salud de la población fundamentalmente campesina desplazada, que estarían más expuestas a padecer de LC ${ }^{(12)}$.

Por su parte, la educación cumple una función fundamental en la prevención y control de la LC, ya que puede ayudar a aportar los conocimientos básicos sobre las medidas de salud que deben desarrollar los habitantes de regiones endémicas; entre éstas se encuentran la recomendación del uso de mallas con repelentes para mosquitos o insecticidas (13); acudir al médico en caso de lesiones en la piel; y el control de los reservorios de la enfermedad, ya sean mamíferos salvajes, como los roedores o domésticos, como el perro ${ }^{(14)}$. Debido a la relación existente entre la educación y el alfabetismo, en la actualidad, el elevado analfabetismo se atribuye a los déficits educativos del pasado; por lo cual al ir mejorando los niveles de educación en dichos Estados, también ha aumentado la alfabetización y con ello, ha disminuido la incidencia de la LC.

La pobreza de ingresos del país conlleva a un aumento de enfermedades infecciosas como la malaria, dengue, Chagas y la LC; lo cual trae consigo la necesidad de crear políticas de salud pública, programas y estrategias que sean económicas, simples, sustentables y localmente aceptables, para mejorar la salud de las poblaciones abandonadas ${ }^{(15)}$. Actualmente, se están desarrollando sistemas de información que pueden ayudar a abordar estos problemas complejos en salud pública con la ayuda de nuevas tecnologías, tales como la satelital ${ }^{(16)}$.

El aumento de la esperanza de vida es subjetivo de las mejoras en las condiciones de salud y calidad de vida de los habitantes de la región, por lo que su relación con el índice de LC es inversamente proporcional (9-11).

El aumento de todos estos indicadores socioeconómicos es el responsable, por consecuencia, del incremento del IDH y de la reducción de la LC durante el período 1994-2003 en dichas regiones. Sin embargo, otros factores no considerados para este estudio pueden estar implicados en los resultados obtenidos, como es el caso de las variaciones climáticas, asociadas al aumento de las tendencias de la Leishmaniosis cutánea, relación observada en el Estado Sucre, Venezuela ${ }^{(17)}$. También, otros estudios muestran como el evento climático conocido como El Niño, aumenta significativamente la tasa de incidencia de la LC en el noreste de Colombia, efecto también demostrado en otros países ${ }^{(18-20)}$.

Comohasidomencionado, losfactoressocioeconómicos, tanto a nivel micro como a nivel macro, deben ser tomados en cuenta, ya que como se ha mostrado en este estudio, pueden estar relacionados temporoespacialmente con la dinámica de comportamiento de la Leishmaniosis cutánea, con una significativa heterogeneidad con respecto al impacto que pueden tener en esta enfermedad tropical, motivo por el cual se hace necesario incrementar este tipo de estudios ecológicos a nivel de países endémicos para esta protozoosis, haciendo evaluaciones comparativas entre diferentes regiones o niveles administrativos y posiblemente incluyendo otros indicadores de desarrollo humano así como marcadores socioeconómicos que pudiesen relacionarse con la enfermedad (21). 
Desde el punto de vista económico, algunos estudios han respaldado el gran impacto de la Leishmaniosis al analizarse su carga en el gasto global del sistema de seguridad social en lugares como la Guyana Francesa donde se ha estimado que el $0,43 \%$ de éste, se consume por consecuencia de esta enfermedad, pese a que no se han logrado medir los días de trabajo suspendidos, los pagos por incapacidades y las hospitalizaciones del personal militar ${ }^{(7,21)}$. Inclusive en algunas regiones de Brasil se han evidenciado importantes efectos socioeconómicos de la Leishmaniosis e impacto de las condiciones sociales y económicas en la epidemiología de esta enfermedad (22).

Podría decirse que los principales efectos de la Leishmaniosis en las regiones endémicas se relacionan con las consecuencias psicosociales y los costos económicos tanto para el sistema de salud estatal como para la familia del paciente; no obstante la importancia de estas consecuencias económicas están de acuerdo con la situación familiar - unidad social que en la mayoría de las veces ha sido solidaria con la atención del paciente. Situación que puede agravarse en los casos de resistencia al tratamiento, indiferencia de las instancias responsables del programa de Leishmaniosis en el estado y un alto grado de pobreza ${ }^{(21)}$.

Por lo anteriormente expuesto se han evaluado vínculos sociales, económicos y psicológicos - como indica el IDH para efectos de esta investigación - con la calidad de vida de las comunidades que residen en focos endémicos. Dichas asociaciones varían según el tipo de enfermedad - acá sólo estudiado para la LC, debido a que representa la mayor carga de morbilidad - y los aspectos que involucra el concepto de calidad de vida a nivel individual como comunitario.

Un punto importante en algunas regiones del presente estudio, como Trujillo, es la consideración sobre el fenómeno de urbanización de la enfermedad. En dicho estado la Leishmaniosis ejerce efectos sobre los diferentes estratos socioeconómicos debido a la urbanización no planificada. Analizando dicho fenómeno, con seguimiento de los cambios poblacionales de los flebótomos y la ocurrencia de nuevos casos urbanos donde en los últimos años se ha intensificado la construcción de viviendas de clase media y alta, se ha comprobado la presencia de flebótomos antropofílicos como Lutzomyia youngi, e infectados con el parásito Leishmania braziliensis junto con marsupiales infectados con $L$. (V.) braziliensis y L. (V.) guyanensis ${ }^{(23)}$.

Consideramos que debe de enfatizarse en la importancia de mejorar las condiciones socioeconómicas para modificar significativamente el comportamiento epidemiológico de la enfermedad en forma positiva así como las condiciones de vida y otras posibles enfermedades tropicales y relacionadas al subdesarrollado que concurrentemente suelen afectar a los pobladores de las zonas rurales de América Latina. La LC propone un desafío particular a los sistemas de salud tradicionales, que requieren de estrategias de intervención dirigidas a las realidades sociales, económicas y ambientales de las décadas presentes y futuras. Es evidente que la indiferencia ante la pobreza genera más pobreza, enfermedad e inestabilidad política llevando a la formación de conflictos por la lucha contra la desigualdad y conllevando a la incontrolable migración. Mientras estos factores persistan la aparición de casos complicados de Leishmaniosis, de nuevos focos endémicos y la ausencia de la adecuada atención médica seguirán aumentando. Asimismo, emerge la necesidad imperiosa de tener en cuenta los conocimientos, creencias, percepciones y prácticas de la población para el estudio de la Leishmaniosis. Además incorporar la participación de la comunidad activamente en las prácticas de prevención e intervención para crear estrategias particulares más efectivas y permanentes en el tiempo ${ }^{(21)}$. De igual forma, lograr que la comunidad conozca su propia realidad es un paso esencial para iniciar cualquier proceso de transformación que contribuya con el desarrollo social, económico, político y cultural de un país basado en el desarrollo humano que conlleva mejor calidad de vida, recordando que éste es el resultado de una inversión en educación y salud - tanto física como emocional que prepara al ciudadano a realizar sus capacidades intelectuales y aprovechar todas las oportunidades a plenitud, que como se observa tiene múltiples efectos en condiciones de salud y enfermedad, dentro de ello la LC.

\section{AGRADECIMIENTOS}

A los profesores Nancy Krieger (Harvard School of Public Health, Boston, EUA) y George Davey Smith (Department of Social Medicine, University of Bristol, Bristol, Reino Unido), por sus orientaciones, comentarios y sugerencias, sobre parte del manuscrito presentado en el $3^{\text {rd }}$ Social Epidemiology Course - European Educational Programme of Epidemiology.

\section{Fuente de Financiamiento}

Se agradece el apoyo de la Compagnia di San Paolo, Turín, Italia, por la beca otorgada a A. J. RodríguezMorales, para la realización del "3rd Social Epidemiology Course - European Educational Programme of Epidemiology", Florencia, Italia, Junio 2007. 


\section{Fuente de Financiamiento}

Autofinanciado.

\section{Conflicto de Interés}

Los autores declaran no tener conflictos de interés en la ejecución y publicación de este artículo.

\section{REFERENCIAS BIBLIOGRÁFICAS}

1. Davies CR, Reithinger $R$, Campbell-Lendrum $D$, Feliciangeli D, Borges R, Rodriguez $\mathbf{N}$. The epidemiology and control of leishmaniasis in Andean countries. Cad Saúde Publica. 2000; 16(4): 925-50.

2. World Health Organization. Communicable diseases 2002: global defense against the infectious disease threat. Geneva: WHO; 2003.

3. Añez N, Nieves E, Cazorla D, Oviedo M, Lugo de Yarbuh A, Valera M. Epidemiology of cutaneous leishmaniasis in Merida, Venezuela. III. Altitudinal distribution, age structure, natural infection and feeding behaviour of sandflies and their relation to the risk of transmission. Ann Trop Med Parasitol. 1994; 88(3): 279-87.

4. Delgado O, Silva S, Coraspe V, Rivas MA, RodriguezMorales AJ, Navarro P, Franco-Paredes C. Cutaneous Leishmaniasis Imported from Colombia to Northcentral Venezuela: Implications for Travel Advice. Travel Med Infec Dis. 2008; 6(6): 376-79.

5. Rodriguez-Morales AJ, Silvestre J, Cazorla-Perfetti DJ. Imported Leishmaniasis in Australia. J Travel Med. 2009; 16(2): 156-57.

6. Hobdell MH, Lalloo R, Myburgh NG. The human development index and per capita gross national product as predictors of dental caries prevalence in industrialized and industrializing countries. Ann NY Acad Sci. 1999; 896(1): 329-31.

7. Dedet JP, Pillot B, Gentilini M. Evaluation of the socioeconomic costs of cutaneous leishmaniasis in French Guiana. Rev Epidemiol Sante Publique. 1991; 39(2): 12933.

8. United Nations Development Programme. Human development report 2006: Beyond scarcity: Power, poverty and the global water crisis. New York: Oxford University Press; 2006.

9. Davies A, Quinlivan G. A panel data analysis of the impact of trade on human development. J Socio Econ. 2006, 35(5): 868-76.

10. Fukuda-Parr S, Shiva Kumar AK. Readings in human development: concepts, measures, and policies for a development paradigm. New York: Oxford University Press; 2003.

11. United Nations Development Programme. Human development report 2004: cultural liberty in today's diverse world. New York: Oxford University Press; 2004.

12. Rodriguez-Morales AJ, López-Zambrano MA, HarterGriep R, Vilca-Yengle LM, Cárdenas R. Aspectos Sociales de la Malaria Importada en Latinoamérica. Rev Peru Med Exp Salud Publica. 2008; 25(2): 208-16.
13. Kroeger A, Avila EV, Morison L. Insecticide impregnated curtains to control domestic transmission of cutaneous leishmaniasis in Venezuela: cluster randomised trial. BMJ. 2002; 325(7368): 810-3.

14. Cardenas $R$, Sandoval $C M$, Rodriguez-Morales AJ, Bendezu H, Gonzalez A, Briceño A, et al. Epidemiology of American tegumentary leishmaniasis in domestic dogs in an endemic zone of western Venezuela. Bull Soc Pathol Exot 2006; 99 (5): 355-358.

15. Franco-Paredes, Jones D, Rodríguez-Morales AJ, Santos-Preciado. Commentary: improving the health of neglected populations in Latin America. BMC Public Health. 2007; 7(1): 11.

16. Rodriguez-Morales AJ. Ecoepidemiología y Epidemiología Satelital: Nuevas Herramientas en el Manejo de Problemas en Salud Pública. Rev Peru Med Exp Salud Publica 2005; 22(1): 54-63.

17. Cabaniel S, Rada T, Blanco G, Rodríguez-Morales AJ, Escalera A. Impacto de los eventos de El Niño Southern Oscillation (ENSO) sobre la leishmaniosis cutánea en Sucre, Venezuela, a través del uso de información satelital, 1994 - 2003. Rev Peru Med Exp Salud Publica. 2005; 22(1): 32-38.

18. Cardenas R, Sandoval C, Rodríguez-Morales AJ, FrancoParedes. Impact of climate variability in the occurrence of leishmaniasis in northeastern Colombia. Am J Trop Med Hyg. 2006; 75(2): 273-77.

19. Cardenas R, Sandoval CM, Rodriguez-Morales AJ, Vivas P. Zoonoses and Climate Variability: the example of Leishmaniasis in Southern Departments of Colombia. Ann N Y Acad Sci. 2008; 1149(1): 326-330.

20. Cárdenas $R$, Sandoval $C$, Rodríguez Morales AJ, Hernández Rangel D, Jaimes E, Mendoza JG. Medio ambiente y protozoosis sistémicas: II. Características fisiográficas del entorno y su asociación en la leishmaniasis visceral. Academia. 2004; 3(1): 35-40.

21. Díaz B. Estudio socioeconómico y epidemiológico de la Leishmaniasis en pacientes del estado Trujillo, Venezuela. [Tesis Doctoral]. Liverpool: School of Tropical Medicine, Liverpool University; 2000.

22. Santos JB, Lauand L, Souza GS, Macedo Vo. Socioeconomic factors and attitudes towards household prevention of American cutaneous leishmaniasis in an endemic area in Southern Bahia, Brazil. Cad Saude Publica 2000; 16(3): 701-8.

23. Scorza JV. Hallazgo de Lutzomyia townsendi (=L. youngi) (Ortiz 1959) naturalmente infectada con Leishmania braziliensis en el área suburbana de Trujillo, Venezuela. Bol Dir Malariol San Amb. 1984; 24(1): 21-28.

Correspondencia: Alfonso J. Rodríguez Morales

Dirección: Sección de Inmunoparasitología, Instituto de Medicina Tropical Felix Pifano, Piso 1, Facultad de Medicina, Universidad Central de Venezuela, Ciudad Universitaria, Caracas, DC 1050, Venezuela.

Teléfono: (58) 212-605-36-47.

Correo electrónico: alfonsorm@ula.ve. 